\title{
Results of an International Postmarketing Surveillance Study of pl-VEGF165 Safety and Efficacy in 210 Patients with Peripheral Arterial Disease
}

\author{
Roman Deev $^{1,2} \cdot$ Igor Plaksa $^{1,3} \cdot$ Ilia Bozo ${ }^{1,4} \cdot$ Artur Isaev $^{1}$
}

Published online: 3 January 2017

(c) The Author(s) 2017. This article is published with open access at Springerlink.com

\begin{abstract}
Introduction The effective treatment of chronic lower limb ischemia is one of the most challenging issues confronting vascular surgeons. Current pharmacological therapies play an auxiliary role and cannot prevent disease progression, and new treatment methods are needed. pl-VEGF165, a gene therapy drug, was approved in Russia for the treatment of atherosclerotic peripheral arterial disease (PAD) after clinical studies in 2011. The study drug is an original gene construction in which pl-VEGF165 $1.2 \mathrm{mg}$ is the active substance.

Objective This postmarketing surveillance study was undertaken to evaluate the safety (identification of uncommon side effects) and efficacy of gene therapy in patients in routine clinical practice.

Methods In total, 210 patients with stage II-III chronic limb ischemia (according to the Fontaine classification modified by AV Pokrovsky) in 33 healthcare facilities in Russia and the Ukraine were enrolled in the study. The control group $(n=60)$ received conservative therapy without prostaglandins and prostacyclins, and the treatment group $(n=150)$ received treatment with pl-VEGF165 as two intramuscular injections for a total dose of $2.4 \mathrm{mg}$.
\end{abstract}

Igor Plaksa

i.plaksa2014@yandex.ru

1 Human Stem Cells Institute, Bld. 2, 3 Gubkina Str., P.O. box 373, Moscow 119333, Russia

2 Ryazan State I.P. Pavlov Medical University, Ryazan, Russia

3 Pavlov First Saint-Petersburg State Medical University, Saint Petersburg, Russia

4 Moscow State University of Medicine and Dentistry, Moscow, Russia
Pain-free walking distance (PWD) (the primary efficacy criterion for Fontaine stages II-III), blood flow linear velocity (BFLV), and ankle-brachial index (ABI) were monitored for 6 months. The safety of pl-VEGF165 gene transfer in terms of the trial protocol was initially evaluated 6 months after the start of the study; adverse events (AEs) and serious adverse events (SAEs) were recorded during both routine visits and unscheduled requests for medical care.

Results Overall, PWD increased by 177\%, from $100.3 \pm 6.9$ to $277.1 \pm 16.2 \mathrm{~m}(p=0.0001)$, in the treatment group, whereas the mean value was unchanged in the control group $(p=0.218)$. Both BFLV and ABI values increased by $24 \%(p=0.0001)$ in the treatment group but decreased in the control group. The greatest therapeutic effect was observed for stage III disease: PWD increased by $683 \%$ ( $p=0.0001$ ). No angiogenic therapy-related AEs or side effects were recorded, and target limb salvage was 96 and $97 \%$ in the treatment and control groups, respectively. The results obtained in this study are not significantly different from those observed in the phase IIb/III registration clinical study completed in 2011.

Conclusion pl-VEGF165 intramuscular gene transfer is an effective treatment for moderate to severe claudication due to chronic lower limb ischemia in routine clinical practice. ClinicalTrials.gov identifier: NCT02369809. 


\section{Key Points}

pl-VEGF165 in combination with standard pharmacological therapy significantly improves clinical signs of claudication in patients with chronic lower limb ischemia in routine clinical practice.

The results of this postmarketing surveillance study showed that the therapeutic effect was most noticeable in patients with stage IIb and III disease.

No angiogenic therapy-related adverse events or side effects were recorded.

\section{Introduction}

Cardiovascular diseases (CVDs) are the third highest nosological group_-preceded only by cancer and hereditary diseases-for which the feasibility of using gene therapy has been widely studied, with 200 registered clinical trials to date [1]. Given the significant role of ischemia in the pathogenesis of most diseases in this group, the therapeutic correction of tissue perfusion via induction of vasculogenesis is a gene therapy application of interest. This is primarily achieved using gene therapy constructions based on viral and/or plasmid vectors with transcription factor and growth factor genes capable of inducing proliferation, migration, and differentiation of cellular elements of the vessel wall, such as endothelial cells and fibroblasts, thus forming new blood vessels of the microvasculature.

Several forms of peripheral arterial disease (PAD), particularly atherosclerosis, lead to stage II-III ischemia (intermittent claudication and critical lower extremity ischemia without necrotic ulceration of the foot) and are the first nosological entities of the CVDs in which a gene therapy approach has been successfully integrated into clinical practice; resulting in the retention of patients for at least 3 years $[2,3]$. The feasibility of using plasmid vectorbased gene therapeutic constructions for these purposes is still under debate. No large studies, such as TAMARIS, TALISMAN-201 (Therapeutic Angiogenesis Leg Ischemia Study for the Management of Arteriopathy and NonHealing Ulcer), and RAVE (Regional Angiogenesis with Vascular Endothelial Growth Factor (VEGF) in peripheral arterial disease), have detected a reliable clinical effect compared with placebo. Therefore, this method may be considered ineffective because of its low transfection and failure to achieve a significant level of target gene expression at the injection site [4-6]. For our study, because no differences have been previously observed with the treatment of critical ischemia with ulcerous-necrotic changes and diabetes mellitus when compared with a control group, we chose a small population of patients with clearly determined indications [4].

The active substance of the study drug is a plasmid with the $V E G F$ gene. The safety, feasibility, and long-term efficacy of pl-VEGF165 were evaluated in a phase IIb/III multicenter randomized trial in 2011 that enrolled 100 patients with intermittent claudication due to chronic lower limb ischemia (stage IIa to III according to the Fontaine classification modified by AV Pokrovsky) for whom reconstructive surgery or endovascular treatment were unsuitable [2]. The indications for its use meet the inclusion criteria of this study.

As results from registration studies sometimes differ from those in clinical practice [7], we examined the safety and efficacy of pl-VEGF165 (Neovasculgen) in 33 healthcare facilities in Russia and the Ukraine (ClinicalTrials.gov: NCT02369809) and compared the results with those from postmarketing surveillance and registration phase IIb/III clinical studies [2, 3].

\section{Materials and Methods}

\subsection{Study Population}

Patients with intermittent claudication and critical lower limb ischemia of atherosclerotic genesis that correlated with stage II-III (a pain-free walking distance [PWD] of $<200 \mathrm{~m}$ and resting pain) according to the Fontaine-Pokrovsky classification (2004) were enrolled in the study $(n=210)$ and randomized into either the control group $(n=60)$ or the treatment group $(n=150)$. Depending on the anatomy and pathological process involved, patients had atherosclerotic lesions of the following types: (1) proximal-primarily occlusion of the iliac-femoral and popliteal arteries; (2) multilevel-occlusions in the iliac-femoral, popliteal, and tibial arteries; or (3) distal-occlusions or hemodynamically significant stenosis in tibial arteries.

\subsection{Description of pl-VEGF165}

The study drug is an original gene construction containing a supercoiled plasmid DNA (1.2 mg)-encoding pCMVvegf165 as the active substance. It is now marketed as 'Neovasculgen' [2]. As stated, the indications for use meet our inclusion criteria. The drug was supplied to the study centers as a sterile lyophilisate that was then dissolved in $2 \mathrm{ml}$ of water immediately before administration as an intramuscular injection. The drug was administered into calf muscles at four to five injection sites in the lower and middle third of the posterior part of the calf. 


\subsection{Study Design and Time Checkpoints}

This was an open, controlled, prospective, comparative, multicentre study design. All patients received standard conservative therapy for chronic limb ischemia without cilostazol and prostaglandins and without surgical or endovascular vessel reconstruction. Surgical and endovascular vessel reconstruction was unsuitable for all of the patients. The study duration was 6 months, with control examination time points upon enrolment and at 14, 90, and 180 days (Fig. 1).

Every patient was informed about the study goals and objectives and the potential benefits and risks of participating prior to signing the voluntary informed consent form. The study protocol was approved by the local ethics committee at every study site. Eligible patients were randomly assigned to either treatment arm using a computergenerated block randomized list (block size 5) for every individual patient using consecutively numbered and sealed envelopes. The sealed envelopes were prepared in advance of the study by one of the researchers, and the local trial coordinator who enrolled patients and assigned them to groups was unable to access the block randomized list. Characteristic of this study design, the participants, surgeons, and local trial coordinator were not blinded to the group assignment.

\subsection{Safety Assessment}

Adverse events (AEs) and serious adverse events (SAEs) were recorded to enable evaluation of drug safety at 6 months. An electrocardiogram (ECG), blood hematology and biochemistry, a coagulation panel, and urinalysis were performed for all patients during screening to detect any concomitant and underlying disorders.

\subsection{Efficacy Assessment}

Given that patients with ulcerous-necrotic changes in limb tissues were excluded from the study, a PWD value was used as the primary efficacy endpoint. This was measured using a treadmill with an elevation angle of $0^{\circ}$ and a speed of $1 \mathrm{~km} /$ $\mathrm{h}$; the walking distance was recorded from the start to pain in the limb muscle (evidence grade B according to the TASC-II [Trans-Atlantic Inter-Society Consensus Document on Management of Peripheral Arterial Disease]). Disease was staged according to the Fontaine classification modified by AV Pokrovsky, which is widely applied by Russian and Ukrainian vascular surgeons. According to this classification, a PWD $>200 \mathrm{~m}$ indicates stage IIa disease, and $<200 \mathrm{~m}$ but $>50 \mathrm{~m}$ indicates stage IIb disease; furthermore, $<50 \mathrm{~m}$ and/ or resting pain without any ischemic tissue changes is the classification for trophic ulcers and/or necrosis.
Fig. 1 Design of the postmarketing surveillance study. $A B I$ ankle-brachial index, $B F L V$ blood flow linear velocity, $P W D$ pain-free walk distance

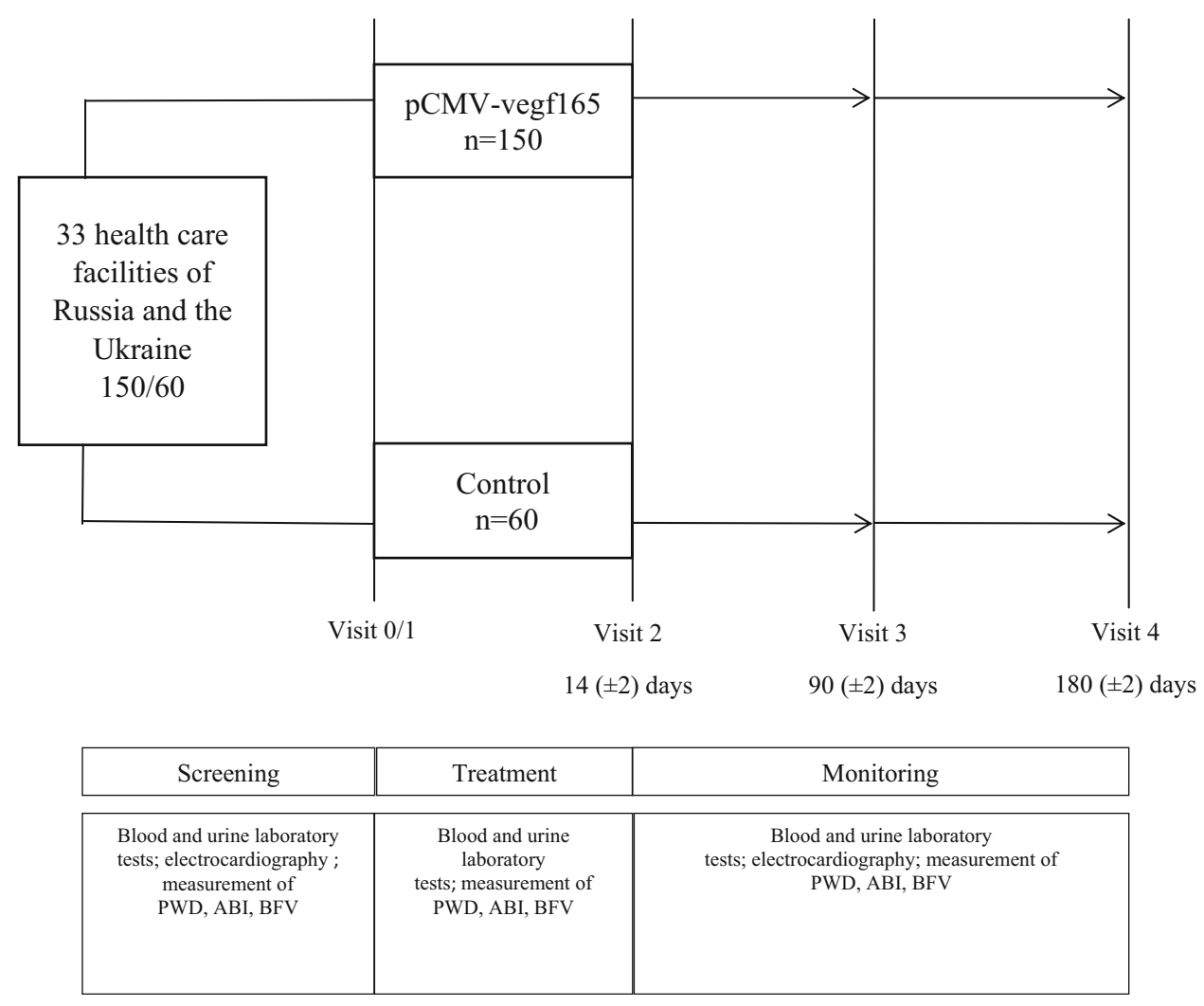


Table 1 Baseline characteristics of postmarketing study subjects

\begin{tabular}{|c|c|c|c|}
\hline Parameter & $\begin{array}{l}\text { Control group } \\
\text { (standard therapy; } n=60 \text { ) }\end{array}$ & $\begin{array}{l}\text { Treatment group } \\
(\text { pl- VEGF165; } n=150)\end{array}$ & $\begin{array}{l}\text { Treatment group (registration } \\
\text { phase IIb/III study; } n=75 \text { ) }\end{array}$ \\
\hline Average age & $68.9 \pm 7.1$ & $62.7 \pm 9.4$ & $67.8 \pm 9.0$ \\
\hline \multicolumn{4}{|l|}{ Sex distribution } \\
\hline M & $44(74.0)$ & $128(85.0)$ & $60(80.0)$ \\
\hline $\mathrm{F}$ & $16(26.0)$ & $22(15.0)$ & $15(20.0)$ \\
\hline \multicolumn{4}{|l|}{ Diabetes mellitus } \\
\hline Type 1 & $1(1.6)$ & $3(2.0)$ & No \\
\hline Type 2 & $5(8.3)$ & $13(8.6)$ & $12(16.0)$ \\
\hline \multicolumn{4}{|l|}{ Smoking } \\
\hline Yes & $32(53.0)$ & $112(74.0)$ & $32(42.6)$ \\
\hline No & $28(47.0)$ & $38(26.0)$ & $43(57.3)$ \\
\hline \multicolumn{4}{|c|}{ Previous revascularization } \\
\hline Yes & $42(70.0)$ & $98(62.0)$ & $41(54.6)$ \\
\hline No & $18(30.0)$ & $52(38.0)$ & $33(45.4)$ \\
\hline \multicolumn{4}{|l|}{ CAD } \\
\hline Yes & $28(46.6)$ & $64(42.6)$ & $28(37.3)$ \\
\hline No & $32(53.4)$ & $86(57.4)$ & $47(62.7)$ \\
\hline \multicolumn{4}{|c|}{ Severity of chronic limb ischemia } \\
\hline IIa & $7(11.0)$ & $11(7.0)$ & $9(12.0)$ \\
\hline IIb & $34(57.0)$ & $79(53.0)$ & $57(76.0)$ \\
\hline III & $19(32.0)$ & $60(40.0)$ & $9(12.0)$ \\
\hline \multicolumn{4}{|l|}{ Level of occlusion } \\
\hline Proximal & $19(32.0)$ & $35(23.0)$ & $38(50.0)$ \\
\hline Distal & $24(40.0)$ & $97(65.0)$ & $16(22.0)$ \\
\hline Multilevel disease & $17(28.0)$ & $18(12.0)$ & $21(28.0)$ \\
\hline PWD, m & $110.3 \pm 3.0$ & $100.3 \pm 7.1$ & $135 \pm 12.2$ \\
\hline ABI, U & $0.51 \pm 0.01$ & $0.49 \pm 0.01$ & $0.51 \pm 0.02$ \\
\hline $\mathrm{BFLV}, \mathrm{cm} / \mathrm{s}$ & $18.2 \pm 0.6$ & $23.9 \pm 2$ & $14.2 \pm 1.6$ \\
\hline
\end{tabular}

Data are presented as $n(\%)$ or mean \pm standard deviation unless otherwise indicated

$A B I$ ankle brachial index, $B L F V$ blood flow linear velocity, $C A D$ coronary artery disease, $P W D$ pain-free walking distance

Secondary efficacy endpoints were blood flow linear velocity (BFLV) of the affected extremity and the anklebrachial index (ABI) (Table 1). Mortality and amputation rates at 6 months were also calculated in both groups.

\subsection{Comparison of Postmarketing Surveillance and Results of the Registration Phase IIB/III Clinical Studies}

A comparative analysis was conducted between the treatment groups in the postmarketing surveillance $(n=150)$ and the registration phase IIb/III clinical studies $(n=75)$ using the primary and secondary efficacy endpoint values $[2,3]$. Baseline mean values for both groups are provided in Table 1.

\subsection{Statistical Data Processing}

Absolute values of the efficacy endpoints (PWD, ABI, and BFLV) complied with a non-normal distribution. Therefore, non-parametric methods (Mann-Whitney $U$ test and Wilcoxon signed-rank test) were used for the statistical analysis.

\section{Results}

\subsection{Safety Assessment}

No AEs related to the use of pl-VEGF165 were recorded. Neither tumor formation nor impaired vision or any other pathology that could indirectly indicate angiogenic therapy 
complications were detected at any follow-up time points in this study. Six patients from the treatment group $(n=150)$ and two patients from the control group $(n=60)$ underwent amputations after progression of critical ischemia. Thus, the amputation-free survival was 96 and $97 \%$ in the treatment and control groups, respectively (Table 2).

\subsection{Efficacy Assessment}

A comparative analysis of the baseline values for each patient group detected no significant differences in sex composition, age, or primary and secondary endpoint values (Table 1); therefore, the groups were comparable. Both groups consisted primarily of patients with distal lower limb artery disease and stage IIb disease (Table 1).

The PWD increased from $100.3 \pm 6.9$ to $191.3 \pm 11.9 \mathrm{~m}(p=0.007)$ in patients treated with plVEGF165 during the first 3 months of the study, and the mean value was $277.1 \pm 16.2 \mathrm{~m}$ by the end of the study, an increase of $176 \%$ ( $p=0.0001)$. Functional state did not significantly change in the control group. The most evident increase in the rate of PWD was observed in patients with stage III disease (critical ischemia): from $24.6 \pm 14.6$ to $188.5 \pm 31.6 \mathrm{~m}(683 \%)$ over 6 months $(p=0.000)$. In patients with stage IIb disease, the PWD value increased from $\quad 104.45 \pm 5.1 \quad$ to $278.08 \pm 19.9 \mathrm{~m} \quad(167 \%$; $p=0.000$ ), whereas patients with stage IIa disease had the

Table 2 Serious adverse events in the treatment and control study groups during 6-month period

\begin{tabular}{lll}
\hline & $\mathrm{pl}-\operatorname{VEGF165}(n=150)$ & Control $(n=60)$ \\
\hline Number of amputations & $6(4 \%)$ & $2(3 \%)$ \\
AMI & 1 & 1 \\
ACVE & 1 & 0 \\
Mortality & 3 & 2 \\
\hline
\end{tabular}

$A C V E$ acute cerebrovascular event, $A M I$ acute myocardial infarction lowest rate of increase: $253.1 \pm 23.4$ to $466.2 \pm 39.5 \mathrm{~m}$ $(84 \% ; p=0.007)$.

The smallest increase in PWD, from $110.7 \pm 14.9$ to $246 \pm 30 \mathrm{~m}(122 \% ; p=0.000)$, was observed in patients with multilevel lower limb PAD. The PWD incremental value was $167.4 \mathrm{~m}(175 \%)$ and $183.5 \mathrm{~m}(185 \%)$ in patients with proximal and distal occlusions, respectively. The PWD values did not change significantly in the control group patients with all types of disease.

If the PWD incremental value of $>30 \%$ is conditionally accepted as a clinically significant effect of pl-VEGF165, the success rate was $75 \%$ with the highest relative rate among patients with stage IIb disease (Fig. 2). More patients with stage III disease experienced a PWD incremental value of $>100 \%$ within 6 months.

The average BFLV increase in the treatment group was $5.8 \mathrm{~cm} / \mathrm{s}$, from $23.9 \pm 2$ to $29.7 \pm 1.7 \mathrm{~cm} / \mathrm{s}(p=0.0001)$, which correlated with improved macrohemodynamics, and the $\mathrm{ABI}$ value increased from $0.49 \pm 0.01$ to $0.61 \pm 0.02$ by the end of the study $(p=0.0001)$. Conversely, the control group exhibited a moderate decrease in BFLV and $\mathrm{ABI}$ values, 3 and $2 \%$, respectively, by the end of the study. The differences in ABI and BFLV values between the groups were already statistically significant at the 3-month time point (Table 3).

\subsection{Comparative Evaluation of the Results of the Postmarketing and Registration Phase IIb/III Clinical Studies}

More patients had stage III disease in the postmarketing study treatment group than in the registration study: 40 and $12 \%$, respectively. Therefore, the average PWD value in the postmarketing study group was significantly lower, by $35 \mathrm{~m}(p=0.004)$. In addition, more patients $(50 \%)$ in the phase IIb/III clinical study had proximal disease, whereas $65 \%$ of patients in the postmarketing study had distal occlusions. There were no other differences between these groups.
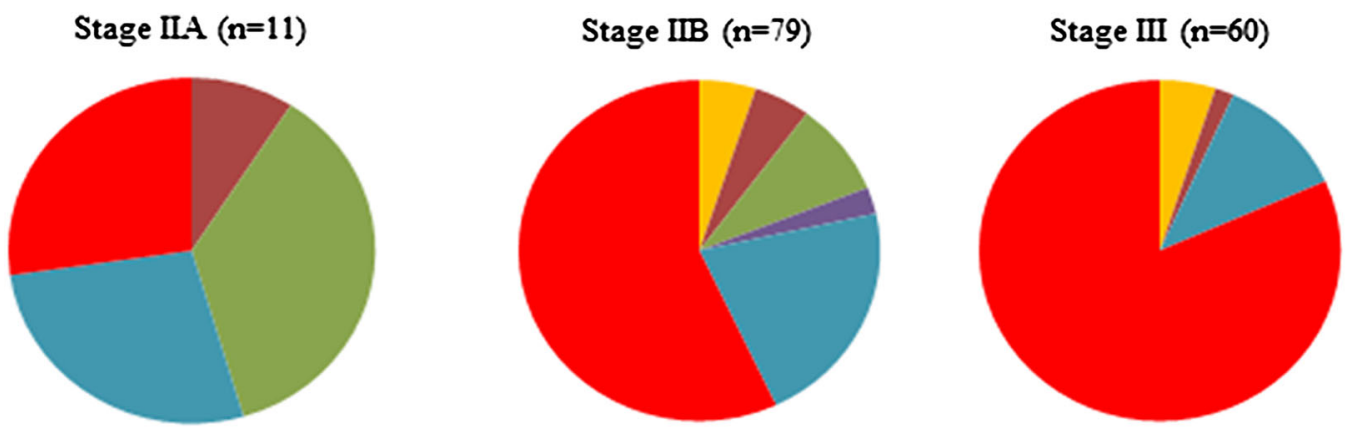

Fig. 2 Proportion of the treatment group patients with a pain-free walk distance increase extent depending on the initial disease stage: red indicates an increase of $>100 \%$; blue indicates an increase of
50-100\%; purple indicates an increase from 30 to 50\%; green indicates an increase $<30 \%$; yellow indicates no increase; brown indicates negative dynamics 


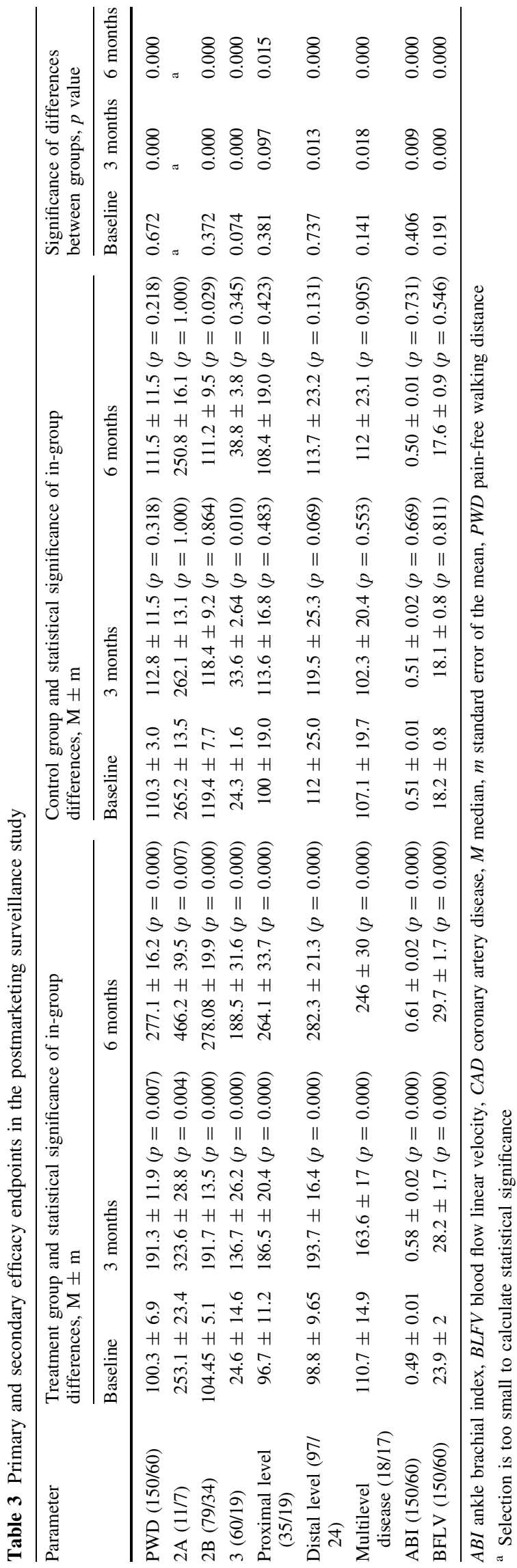

The use of pl-VEGF165 resulted in a statistically significant increase in PWD: from $100.3 \pm 6.9$ to $277.1 \pm 16.2 \mathrm{~m}(177 \%)$ and from $135.3 \pm 12.2$ to $284.7 \pm 29.8 \mathrm{~m}(110 \%)$ in the postmarketing and phase IIb/III clinical studies, respectively. In-group differences became statistically significant in both groups at the 3-month time point. As the PWD incremental value was more pronounced in the postmarketing study treatment group, its baseline difference became balanced at 6 months, i.e., at that time the average PWD value was $277.1 \pm 16.2 \mathrm{~m} \quad(p=0.0001) \quad$ and $\quad 284.7 \pm 29.8 \mathrm{~m}$ $(p=0.0001)$ in the postmarketing and phase IIb/III clinical studies, respectively. A comparative and selective evaluation of the therapeutic effect in both studies indicated similar tendencies: patients with stage III disease had the most noticeable incremental PWD rate, and this increase was significantly higher in these patients in the postmarketing treatment group (683\%) than in the registration clinical study (236\%).

Because there were no significant between-group differences in average ABI values at the start of the study, a more noticeable positive parameter shift was observed in the postmarketing study group (24 vs. $9 \%$ in the phase IIb/ III clinical trial). There was a statistically significant increase in ABI values, 24 and 59\%, in both studies (Table 4).

\section{Discussion}

The objective of this postmarketing surveillance study was to evaluate the safety and efficacy of pl-VEGF165 in clinical practice. The treatment results for 210 patients at 33 study sites confirmed that pl-VEGF165 was well tolerated and not accompanied by drug-related AEs for at least 6 months. The introduction of pl-VEGF165 in a combined treatment resulted in a significantly improved functional state for patients with stage II-III chronic limb ischemia, which manifested as an increase in average PWD of $177 \%$ at 6 months and correlated with positive changes in instrumental diagnostic parameters.

Only the TAMARIS study included more subjects and study sites (i.e., 525 patients with critical limb ischemia of multiple etiologies treated with a plasmid containing a gene encoding fibroblast growth factor [FGF]) [4]. TAMARIS included patients with end-stage disease, so amputation rate was determined to be a stringent efficacy endpoint; however, results for this endpoint did not differ from those in the control group. It could be assumed that gene therapy is less efficient in end-stage disease accompanied with ischemic defects and the threat of amputation; however, as other studies have shown this strategy to be effective [8], whether treatment earlier in the disease may be more 


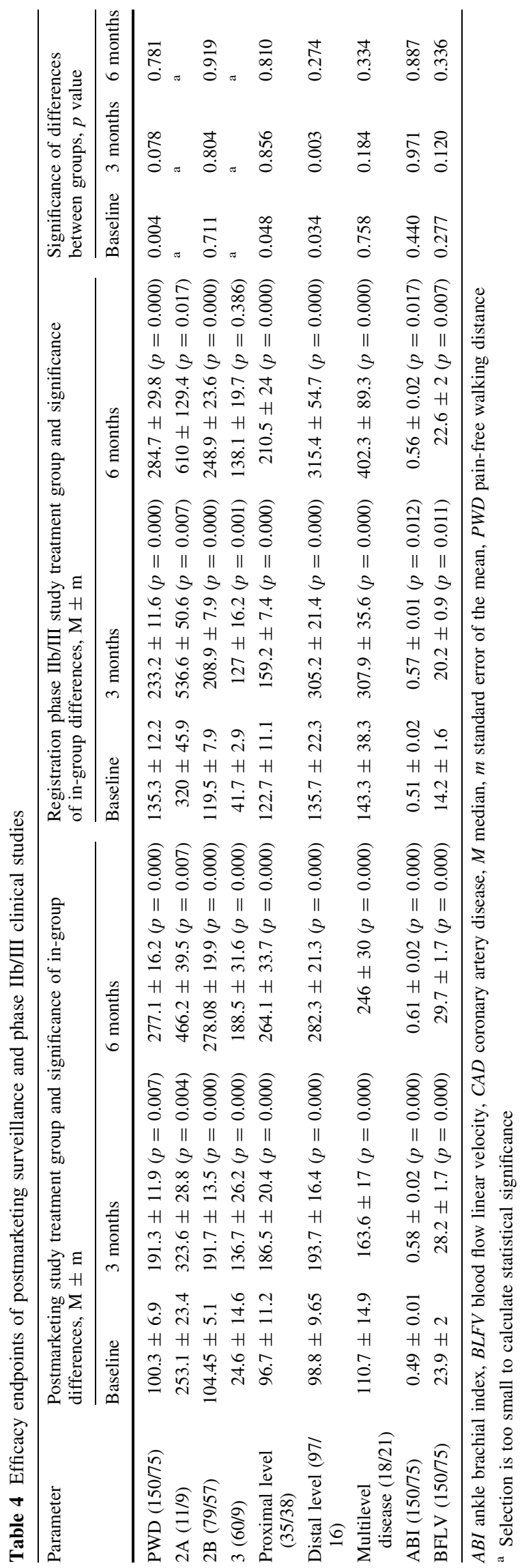

appropriate is unknown at this point, and results may depend on the agent and method of delivery.

Given the severity of critical limb ischemia and the low probability of a therapeutic effect from angiogenic therapy on disease prognosis, only patients with earlier chronic limb ischemia (intermittent claudication) were included in this study and many others. A double-blind 13-month study recruited 289 subjects treated with an adenovirus-based gene therapy drug with a hypoxia-inducible factor-1 (HIF1) gene; PWD was also the primary efficacy endpoint [9]. This study indicated that gene induction of angiogenesis via a local increase in endogenic HIF-1 synthesis at the intermittent claudication stage was followed by a significant increase in PWD in the treatment versus placebo groups, which correlated with the ABI values. Thus, therapeutic angiogenesis at the mid-term stages of disease is more likely to prevent the progression of ischemia; our study confirms this.

Despite numerous studies of angiogenic therapy for PAD, pl-VEGF 165 continues to be the only gene therapy employed for the treatment of chronic limb ischemia in Russia and the Ukraine. However, a number of drug candidates are undergoing phase III clinical studies, one of which is a hepatocyte growth factor (HGF) plasmid known as AMG0001. Two placebo-controlled clinical trials $[10,11]$ in 131 subjects indicated this gene therapeutic construction provided a statistically significant reduction in ulcerous defect area in patients with critical limb ischemia, in correlation with increased transcutaneous oxygen $\left(\mathrm{tcpO}_{2}\right)$ and $\mathrm{ABI}$ values, versus placebo. When planning the phase III study, the investigators selected 'time to major amputation after angiogenesis induction' as the primary efficacy endpoint because the study included patients with the most severe disease, in whom a successful treatment outcome was not expected. This could also be due to their consideration of the TAMARIS study experience, as this efficacy endpoint is less stringent than amputation rate; however, it is useful in the achievement of a significant difference versus placebo. Thus, we assume this gene therapy drug would be registered and introduced into the multifaceted treatment of patients with critical limb ischemia should a statistically significant increase in limb salvage time be observed.

A plasmid developed by ViroMed that expresses two HGF isoforms is another candidate in gene therapy drugs, and a phase III clinical study has been approved by the US FDA (http://www.businesswire.com/news/home/201508 31006330/en/FDA-Approves-Initiation-ViroMeds-VM202Phase-III). Clinical studies demonstrated this drug resulted in a significantly increased number of healed skin ischemic defects in patients with critical limb ischemia. Among the phase II study subjects, $60 \%$ had diabetic foot, for which the use of gene therapy was most effective, and the 
investigators decided to change the inclusion criteria for the phase III clinical study in favor of patients with ischemic diabetic foot syndrome [12]. The study is designed as a multicentre placebo-controlled study due to start in 2016. The drug will be administered as an intramuscular injection in the lower leg, and the primary efficacy endpoint will be the extent of ulcer defect dynamics. However, our study has demonstrated that the selection of patients with advanced disease creates unfavorable conditions for therapeutic angiogenesis, and great care is required when determining the exact patient population that could benefit from plasmid-based gene therapy.

Although the open-label use of study drugs is a limitation, this study was randomized and involved multiple centers ( $>30$ in two countries), and patient enrollment decisions were made independently by a team of vascular surgeons and radiologists on the basis of angiographic results, disease history, previous procedures, and concomitant pathology. Therefore, we suggest these data can be generalized, particularly to patient populations with intermittent claudication.

\section{Conclusions}

The use of pl-VEGF165 in combination with standard pharmacological therapy significantly improves clinical signs of claudication in patients with chronic lower limb ischemia. The international postmarketing surveillance study indicated that gene therapy is as efficient in routine clinical practice as it was in the phase IIb/III registration clinical trial in patients with moderate to severe claudication due to stage IIa-III atherosclerotic chronic lower limb ischemia. Therapeutic effect was most noticeable in patients with stage IIb and III disease. No angiogenic therapy-related AEs or side effects were recorded. However, further studies enrolling more patients are needed to completely evaluate the effects of pl-VEGF165 gene transfer in patients with pain at rest due to peripheral atherosclerosis or autoimmune disorders and patients undergoing peripheral arterial revascularization.

\footnotetext{
Acknowledgements The authors thank the clinical investigators for their contributions to this trial and for participating in the studies.

This international postmarketing surveillance study was conducted according to the Declaration of Helsinki of the World Medical Association Recommendations Guiding Physicians in Biomedical Research Involving Human Subjects (1964, 2000), Rules of Good Clinical Practice in the Russian Federation OST 42-511-99, ICH GCP rules, and valid regulatory requirements.
}

\section{Compliance with Ethical Standards}

Funding This study was funded by the Human Stem Cells Institute OJSC, Moscow, Russia.
Conflict of interest A Isaev, I Bozo, R Deev, and I Plaksa are employees of the OJSC Human Stem Cells Institute. A Isaev holds shares in the Human Stem Cells Institute OJSC.

Open Access This article is distributed under the terms of the Creative Commons Attribution-NonCommercial 4.0 International License (http://creativecommons.org/licenses/by-nc/4.0/), which permits any noncommercial use, distribution, and reproduction in any medium, provided you give appropriate credit to the original author(s) and the source, provide a link to the Creative Commons license, and indicate if changes were made.

\section{References}

1. Rincon MY, Vanden D, Chuah MK. Gene therapy for cardiovascular disease: advances in vector development, targeting, and delivery for clinical translation. Cardiovasc Res. 2015;108(1):4-20.

2. Deev R, Bozo I, Mzhavanadze N, et al. pCMV-vegf165 intramuscular gene transfer is an effective method of treatment for patients with chronic lower limb ischemia. J Cardiovasc Pharmacol Ther. 2015;20(5):473-82.

3. Deev RV, Kalinin RE, Chervyakov YuV, et al. Long-term results of pl-VEGF165 intramuscular gene transfer in patients with atherosclerotic chronic lower limb ischemia. Cardiol Cardiovasc Surg. 2015;4:43-9.

4. Belch J, Hiatt WR, Baumgartner I, et al. Effect of fibroblast growth factor NV1FGF on amputation and death: a randomised placebo-controlled trial of gene therapy in critical limb ischemia. Lancet. 2011;377:1929-37.

5. Nikol S, Baumgartner I, Van Belle E, et al. TALISMAN 201 Investigators. Therapeutic angiogenesis with intramuscular NV1FGF improves amputation-free survival in patients with critical limb ischemia. Mol Ther. 2008;16:972-8.

6. Rajagopalan S, Mohler E, Lederman R, et al. Regional angiogenesis with vascular endothelial growth factor (VEGF) in peripheral arterial disease: design of the RAVE trial. Am Heart J. 2003;145:1114-8.

7. Flather M, Delahunty N, Collinson J. Generalizing results of randomized trials to clinical practice: reliability and cautions. Clin Trials. 2006;3:508-12.

8. Baumgartner I, Pieczek A, Manor O, et al. Constitutive expression of phVEGF165 after intramuscular gene transfer promotes collateral vessel development in patients with critical limb ischemia. Circulation. 1998; 31;97(12):1114-23.

9. Creager MA, Olin JW, Belch JJ, et al. Effect of hypoxia-inducible factor-1alpha gene therapy on walking performance in patients with intermittent claudication. Circulation. 2011;124(16):1765-73.

10. Powell RJ, Goodney P, Mendelsohn FO, et al. HGF-0205 Trial Investigators. Safety and efficacy of patient specific intramuscular injection of HGF plasmid gene therapy on limb perfusion and wound healing in patients with ischemic lower extremity ulceration: results of the HGF-0205 trial. J Vasc Surg. 2010;52(6):1525-30.

11. Powell RJ, Simons M, Mendelsohn FO, et al. Results of a doubleblind, placebo-controlled study to assess the safety of intramuscular injection of hepatocyte growth factor plasmid to improve limb perfusion in patients with critical limb ischemia. Circulation. 2008;118(1):58-65.

12. Henry TD, Hirsch AT, Goldman J, et al. Safety of a non-viral plasmid-encoding dual isoforms of hepatocyte growth factor in critical limb ischemia patients: a phase I study. Gene Ther. 2011;18(8):788-94. 